\title{
Implementation of GSM Based Security System with IOT Applications
}

\author{
Seelam Vasavi Sai Viswanada Prabhu Deva Kumar \\ ITM University, Gwalior (MP), India \\ E-mail: vasavisai9999@gmail.com \\ Shyam Akashe \\ ITM University, Gwalior (MP), India \\ E-mail: shyam.akashe@itmuniversity.ac.in
}

\begin{abstract}
An effective security system is designed and implemented through the application of embedded systems and the Internet of Things (IoT). The main goal of this work is to present the IoT system and modern technology systems / embedded intelligent networks. IoT is the technology of the future in contact machine / machine device / machine is possible thanks to the electronics, sensor technology, software and connectivity system to enable these items to collect and exchange data. Embedded system is a real-time mainframe structure on a particular task, an automatic or electrical system including, often within the limits of real-time raking as sensors, modules with microcontrollers, etc...This is integrated with sensor main server and GSM global system For mobile), it is able to send data and IP addresses through SMS message. This will facilitate the identification of the unknown person embedded in realtime in these devices. The video data are transferred to another back-end server via TCP (Transmission Control Protocol) of the device. This server collects data and provides HTTP Web. A browser is used to view data and can be remotely controlled. This type is giving an idea about the probability and effectiveness of the system, this thesis presents the results of the investigation into the security system and some realistic implementations.
\end{abstract}

Keywords-Atmega16, GSM Module, Passive infrared Sensor, Embedded C, Internet Protocol (IP).

Tools - CodeVisionAVR, PCB Wizard

Programmer-USBasp

\section{INTRODUCTION}

Integrated intelligence is a mechanism by which a specific application device as a combination of microcontroller, protection sensor with integrated signal status and distribution channels of the signal communication module control management intelligent systems, they refer to other technologies with similar convenience in the appearance of the machine / device communication machine / device. Integrated information system is present in many different devices and areas such as health divisions, agriculture, transport, defense and performance. IoT controllers use different communication protocols over the Internet. Aspire of this work is to provide a security system where they connect with each other, with the IoT development in the modern era. In this work, we use a microcontroller (AVR-RISC processor Alf and Vegard), PIR (passive infrared sensor), a GSM module and a smart phone to start an idea. The idea, focusing on the need to design a security system that is moderately tangible. For example, if a family goes to Hometown or wherever you leave your room / house, it must be something that keeps control of the smart home. If someone inside the house should be a device that will make you alerts, like to take pictures, record videos. The best competition of these efforts is a security system through intelligent IP device to access the Internet of things everywhere protocol.

We want to know about the monitoring system that has linked the attachment toward the Internet used for distant screening. During earlier period, safety systems must be monitor through a guardian who was protected during a day by every surveillance room that would be monitored to ensure nothing. It is very important now to have days; the prospect of our remote security system is ease to notice in real time. This system of progressing a message directly to the mobile phone from the cell to facilitate, detect that your surveillance camera capture an incident. This system protects against theft. By being able to see your DVR over the internet, you are very happy to immediately stop a crime.

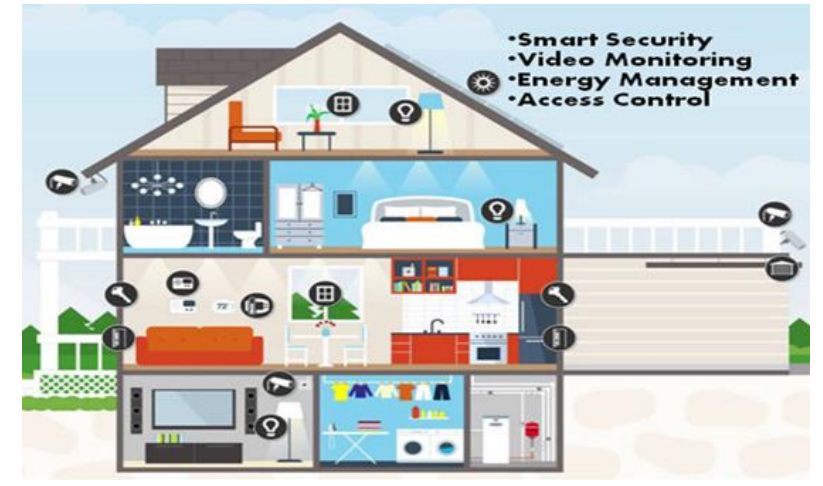

Fig.1. IoT Based Smart Protection 
To operation of each module / component / tool is given below:

\section{8-BIT MICROCONTROLLER}

In this thesis, we use the ATMEGA16L controller is an 8-bit microcontroller designed to support low-power CMOS technology in the high-performance RISC AVR improved structural design $32 * 8$ general functions of the register. It is manufactured by Atmel with high compactness of non-volatile storage disks. On-Chip InSystem Program Flash memory car 16KB, 512KB EEPROM also $1 \mathrm{~KB}$ internal SRAM. This microcontroller has 40 pins, 32 pins are programmable input lines ( 4 ports each port 8 pins) has four channels of pulse width modulation, eight-channel ADC with 10 bits, the 32 lines of output 3 Pines input is used for DC power (5V), 2 Pins used for the crystal oscillator (XTAL2, XTAL1) to 3-pin to AREF, AVCC and RESET. This microcontroller runs at $2.7 \mathrm{~V}-5.5 \mathrm{~V}$ with a power of $0.35 \mathrm{~mA} 1.1 \mathrm{~mA}$ in active mode in idle conditions with a level of $0-8 \mathrm{MHz}$. The software of the boot flash area is still running while the application's flash area is updated to read true while writing. The combination of a programmable automatic flash 8-bit RISC CPU on a monolithic chip system. ATMEGA16L is the most commonly used profitable microcontroller and easy to implement the integrated application. It supports encoding as embedded $\mathrm{C}$, assembler.

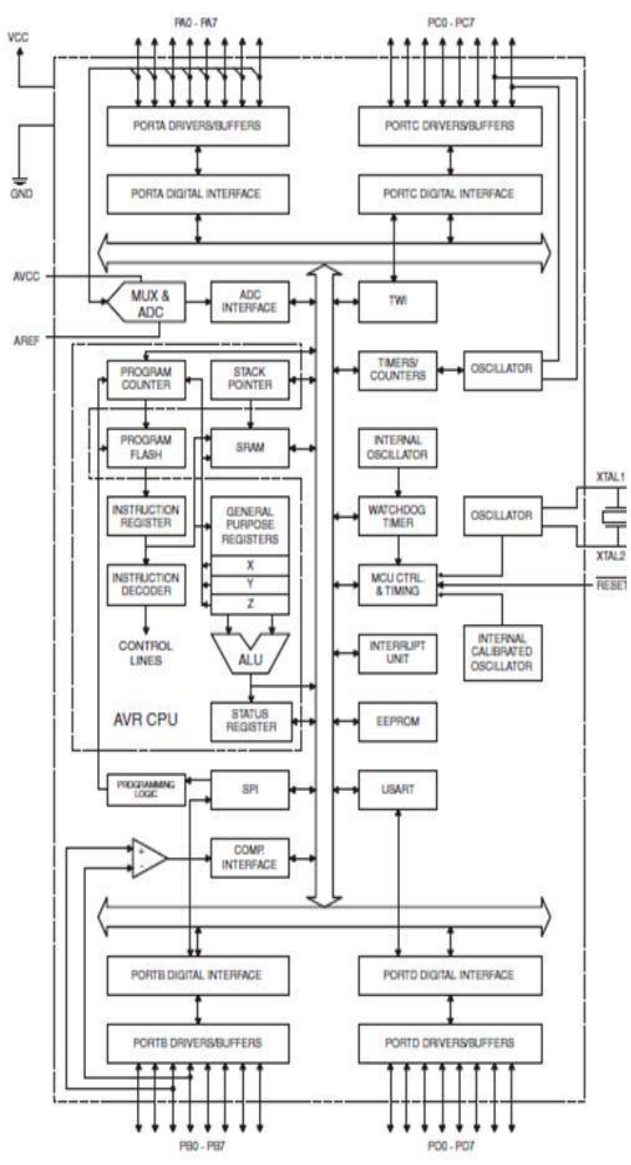

Fig.2. Block Diagram of Atmega16L

\section{GSM MODULE (SIM900A)}

GSM module is used to send / receive messages and make / receive calls as a mobile phone a SIM card from network source. This system done when GSM module connects to the AVR, and then a SIM card provides a link from a server's GPRS cover. The module uses the SIMComm function. It is be able to communicate easily with the module through AT commands. GSM library contains several methods of communication with the module. The GSM modem can work with any GSM network operator SIM card like a mobile phone with its own unique telephone number. The advantage of this modem is the RS232 interface can be used to communicate and develop integrated applications. Control applications such as SMS, data transmission, remote monitoring and recording can be easily developed with this. The modem can be connected directly to the serial port of the PC or to a microcontroller via MAX232. It can be used to send / receive SMS and make / receive voice calls. It can also be used in GPRS mode to connect to the Internet and run many recording applications and data control. GPRS mode can also connect to an FTP server and files for data download this GSM modem registration is a GSM Modem SIM900A Quad-band GSM modem and run very flexible for easy integration with direct applications. It supports functions like voice, SMS, data / fax, GPRS and integrated TCP / IP stack. Up to a mobile network can be connected; the module requires a SIM card provided by a network provider.

It is a wireless module that is not very compact and reliable. SIM900A is a dual GPRS / GSM full-band SMT module that can be integrated into client applications, which allows you to take advantage of small size and cost-effective solutions. With the SIM900A interface provides industry standard GSM / GPRS 900 / 1800MHZ for voice, SMS, data and fax in the form of a small factor and with low power consumption. With a simple configuration of $24 \mathrm{~mm} \times 24 \mathrm{~mm} \times 3 \mathrm{~mm}$, SIM900A can be used in almost all space requirements in their application, especially for the application of slim design and small size.

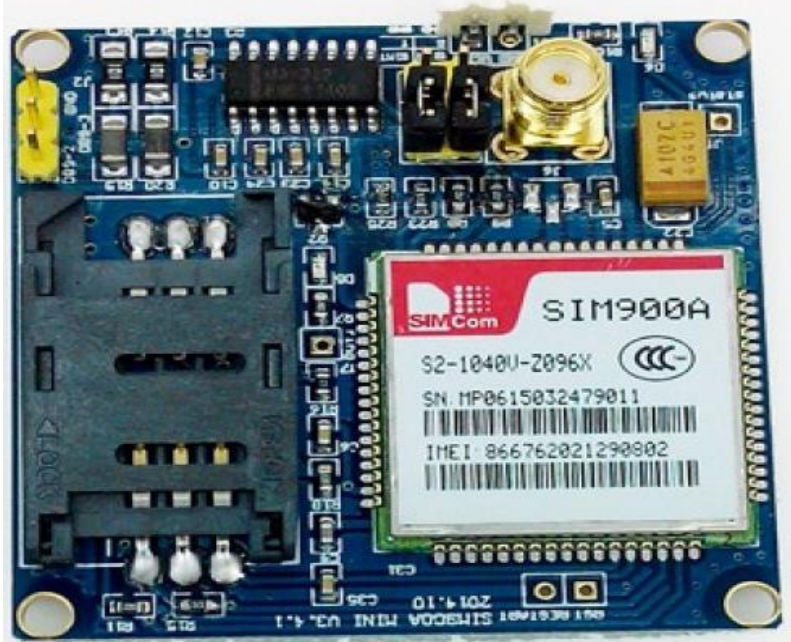

Fig.3. SIM900A GSN Module 


\section{PASSIVE INFRARED SENSOR}

PIR sensors capture the motion allows to sense movement, constantly to prove if a human being moved in or out from the sensor area. They are tiny, low power, cheap, uncomplicated to utilize. For this reason are often found in devices in households and businesses. It also referred to as PIR sensors, "PIR", "pyroelectric" or "IR motion."

For the most part, PIR modules contain 3-pin connector on the surface or underside. The pin can differ among the module triple the pin! It is frequently screened taking place at the exact side of compound a pin, another signal of the end will be energy. The energy input is generally $3-5 \mathrm{VDC}$ but can be as high as $12 \mathrm{~V}$. Sometimes larger elements do not contain direct access and alternative simply run a relay in this case; no land, food and two switch connections.

The output relay can be an "open collector" - that is, it needs a pull-up resistor. If you do not get an uneven output make certain to connect a $10 \mathrm{~K}$ pull-up among the signals and power pins.

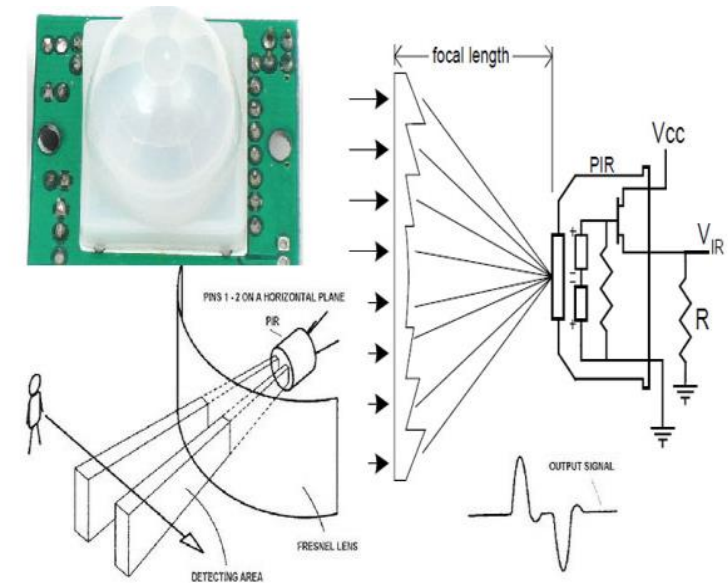

Fig.4. Working of PIR Sensor

\section{EMBEDDED C}

During the years of microprocessor-based systems, programs have been developed, for the use of counters and fused into the EPROM. There was no mechanism to find the program was carried out. They have checked LEDs, switches, etc., the proper implementation of the program. A small number of developers have in circuit emulator (ICE), although those are very expensive and not much consistent. Over moment, the utilize of certain set of processor systems by a program language has been compact for Embedded $\mathrm{C}$ that reduces the integrated choice of programming language and moves. Programming language $\mathrm{C}$ is the most commonly used / integrated controller processors. The arrangement is also used, in particular, however, to implement parts of the code wherever the timing, efficiency code extent etc. They are very important.

Because the assembler programs are explicit headed for processor assembler language, there was no convenient between the structures. To prevail over this drawback, a number of high-ranking languages, include $\mathrm{C}$, emerged. Further languages such as PLM, Modula-2, Pascal, etc. as they came other than not widely accepted. Among them, $\mathrm{C}$ has gained extensive recognition, not just for embedded systems, but in addition for office applications. Although $\mathrm{C}$ has mislaid its luster like a main language for universal applications, and yet it have a physically powerful in embedded encoding. Because of the great recognition of $\mathrm{C}$ in embedded classification, they came across a variety of types of supporting tools such as compiler and cross-compiler, ICE, etc. All this has made the development of embedded systems easier with $\mathrm{C}$. The assembler language seems more embedded for programming Systems to be an obvious choice. The use of assembler language, however, develops on limited effectiveness in terms of code size and speed. In addition, the assembler code leads to higher costs of software growth in addition to code convenient is not present. The development of undersized codes is not a big difficulty; however important programs/task is getting more and more complex to handle in assembler language. Find high-quality programmer assembly, it has also turn to tricky. Therefore, the advanced language is chosen for embedded programming systems. Embedded C utilizes the majority of the syntax and semantics of the $\mathrm{C}$ standard, for example the function main (), the definition of variables, the declaration of the data type, the conditionals (if, switch statement), the trailing (while, for) functions, the arrays and strings, set, bit operations etc...

Note:

1. Compare with assembler language, written by $\mathrm{C}$ Code is further consistent, scalable more convenient among different platforms.

2. C compilers are currently used for practically each and every one of the embedded campaign and currently many experienced programmers in $\mathrm{C}$.

3. Unlike the arrangement, $\mathrm{C}$ has the advantages of CPU independence, rather than specific microprocessors / microcontrollers or systems. It allows users to more easily develop programs that can run on most systems.

\section{INTERNET PROTOCOL}

Internet Protocol (IP) is the most important communication etiquette within the Internet protocol set for passing datagram's crosswise system limitations. The routing task allows the interconnection as well as effectively provides the Internet. IP has the mission of providing packet as of the basis host to the target host exclusively on top of the basis of the IP address in the package headers. In favor of this function, the IP packets define encapsulated configuration of information to be output. In addition, it classifies addressing technique to use the datagram's with source and destination information to be used. Based on the principle of the ultimate Internet protocol design. The network 
infrastructure is fundamentally reliable for each link or node in the link and the transport medium is dynamically connected, the availability. There is refusal measuring or observing services of a central facility or the following network status. To reduce the complexity of the network, the intelligence in the network, is conscious endpoints. The active environment of the Internet with the variety of its apparatus does not assurance to an exacting lane is in point of fact to be able to perform the requested data from the execution or suitable for this, still if the lane is existing and consistent. A scientific constraint is the amount of the information packets allowed on a particular connection. Relevance has to ensure the proper use of the transfer characteristics. This responsibility is also part of the high-level agreement. You can use the (MTU) and path MTU to find the full projection route to the destination. IPv4 link layer can automatically segment the original data into a smaller unit for transmission. In this case, doing some sort of IP fragmentation has been passed in sequence.

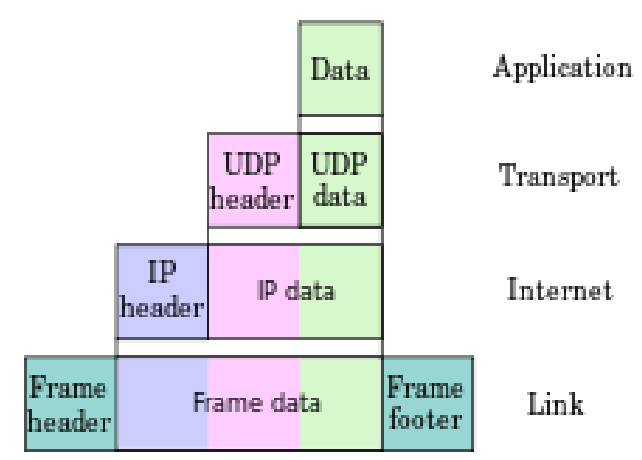

Fig.5. Encapsulation of Application Data from UDP to a Link Protocol Frame

\section{CODEVISIONAVR}

This application works with Windows XP, Vista, Windows 7, Windows 8, and Windows10.32 and 64-bit. Easy integration in the development environment compiled with the ANSI C editor, with automatic indentation, syntax from $\mathrm{C}$ and assembly AVR function parameters, structural elements / fully automated union compatibility. In addition to its own IDE integrates Atmel Studio's 6.2 and 7 CodeVisionAVR.

\section{(A)Compatible Data Types:}

1. Bit, char, int, short, long, long 64-bit float.

2. Library quickly improves floating-point hardware multipliers and instructions for all new ATmega chip cores.

\section{(B) AVR Specific Extensions for:}

1. Transparent and easy access to storage areas EEPROM and Flash memory without special functions and other compilers AVR.
2. Access level bit register I / O

3. Interrupt support

4. General register (I / O) of the new chip (ATtiny2313, ATmega48 / 1688, ATmega165 / 169/325/3250/329/3290/645/649/64) I / GPIOR), ATmega1280 / 2,560,002,181 / 640/256, ATmega406)

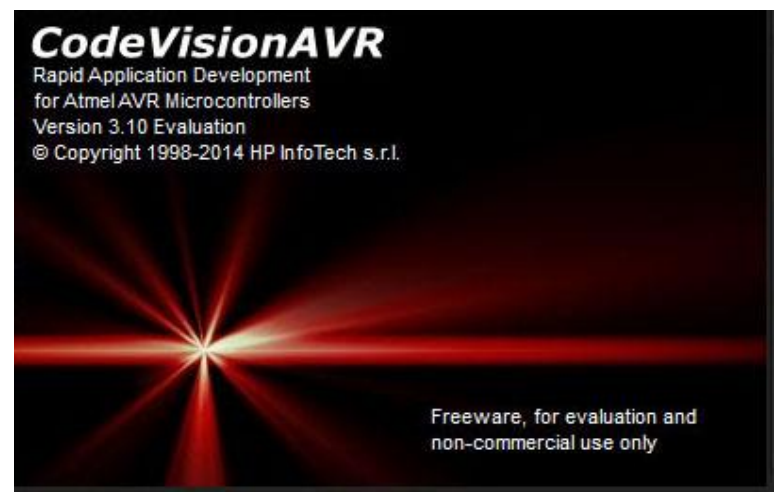

Fig.6. CodeVisionAVR

\section{PCB WIZARD}

PCB Wizard is a powerful package designed for single-sided PCB and both sides (PCB), PCB. Create a circuit diagram, detection circuit, component layout, automatic routing, reporting and data generation material manufacturing, which provide a variety of tools, including all the steps of traditional circuit board production. In addition, the PCB Wizard provides many new and intelligent features that eliminate the steep learning curve typically associated with PCB packages.

The light application comes into the system, but that does not mean it lacks functions. It is a feature-rich application that will help you design. The lab also has tremendous functionality and developers use to see design. There are many display options in this application that means you can see the design from all sides.

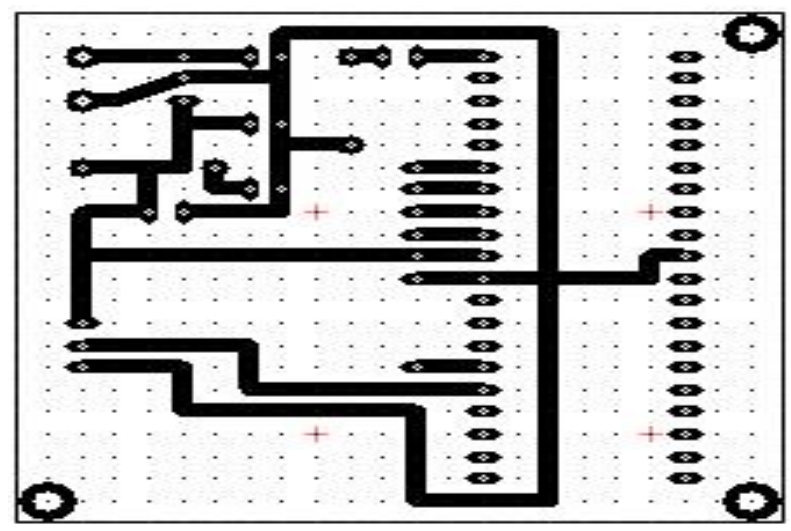

Fig.7. PCB Design in Art World 


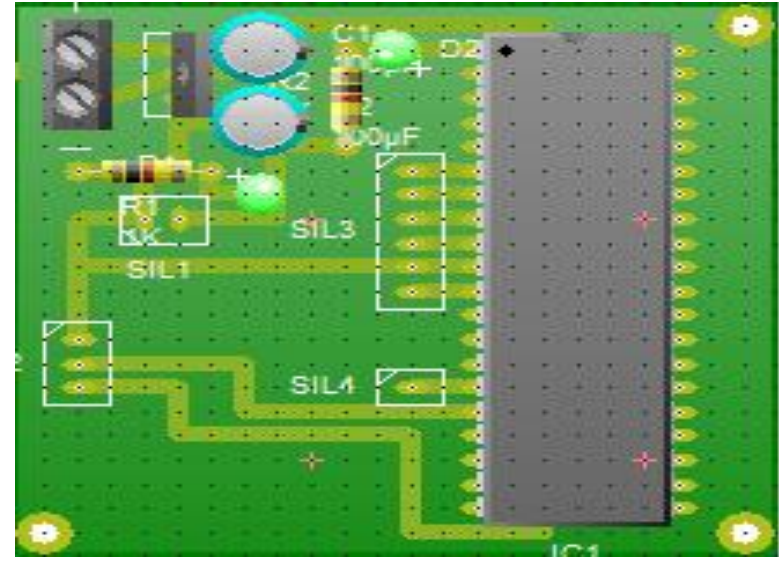

Fig.8. PCB Design in Real World

\section{USBASB PROGRAMMER}

USBasp is an integrated USB controller circuit for Atmel AVR-based controllers. It consists only of one or ATmega88 atmega8 and a pair of passive components. The programmer uses a USB controller firmware only; a special USB driver is not required. To use, this programmer must be installed in USBASP Windows drivers. The power comes really USB programmers, which could be very few large power capacity the USB drive could be damaged. In general, more current $100 \mathrm{~mA}$, the target card must be provided with a separate source. By manipulating bridges fig. 8 the programmers can configure different energy options. Programming must ensure that the programmer can speak with support for software and chip targets. To do this, you can perform read operation (read backup or flash AVR) support software. If the operation is successful, it means that the programmer can communicate with the supporting software and connect it to the target board. If it fails, you must find the causes. The programmer works much more stably when the bridge "CLOCK SLOW" (<to $1.5 \mathrm{MHz}$ ) is short-circuited. If models oscilloscope 062 programming should be used (06201P, 06201KP or 06202KP). The pin assignment of this head is compatible with the programming connection of oscilloscopes.

However, in order to load the microcontroller program (hex file) with the programmer USBASP, you need the appropriate software. Fortunately, there is a free open source program to download, download or manipulate the Flash, EEPROM and AVR microcontroller fuses over the programmer. It is part of the software packages. You can easily install the Sinaprog or eXtreme burners, which has a number of software development tools for Atmel AVR code open.

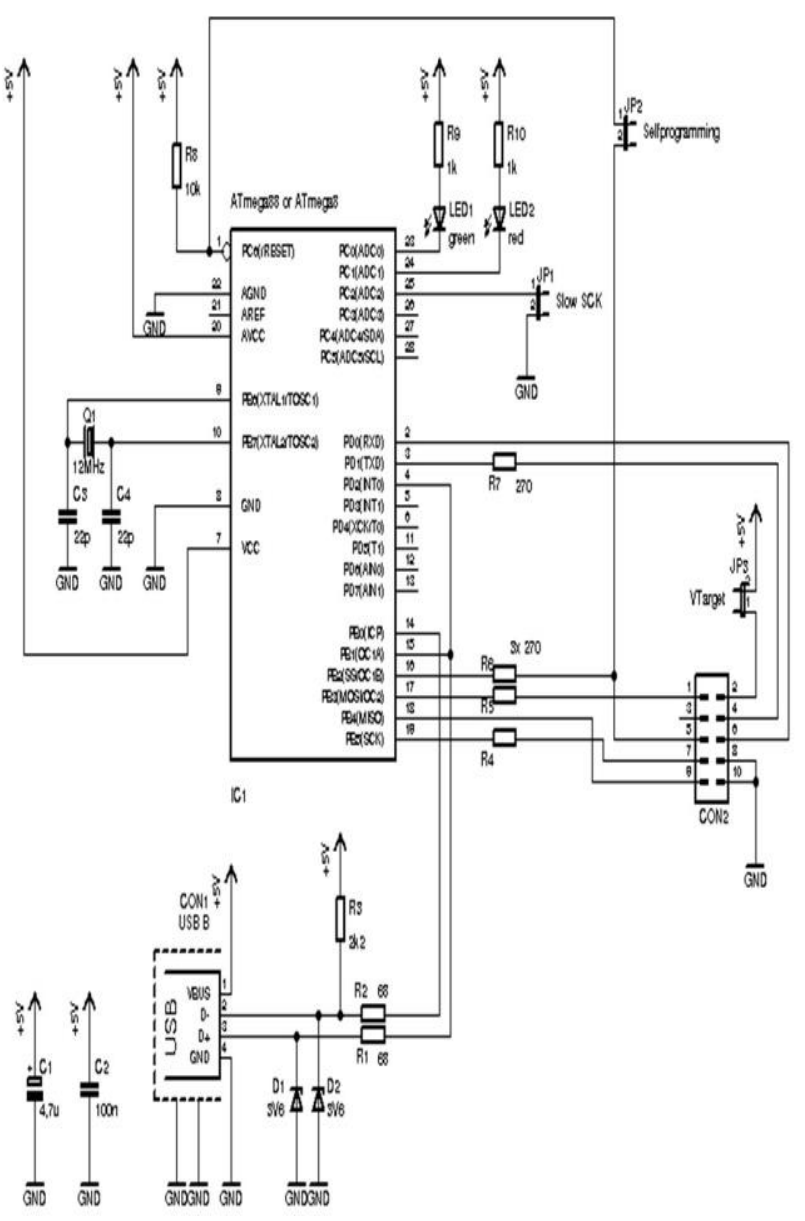

Fig.9. USBasb Programmer Schematic Diagram

\section{SYSTEM SETUP}

\section{A. Installing the Designed Circuit Board}

This configuration requires a microcontroller-designed card, an open-source sensor that directly matches the circuit board, the GSM module (SIM900A) is inserted with the Tx ATmega16 pin PD1 and GSM SIM (2G / 3G) as shown in the figure. The pins MOSI, MISO, SCK, RST, VCC and GND are connected programmers usbasb is en route for loading the hex file to the microcontroller. The entire circuit requires $5 \mathrm{vdc}$ with $110 \mathrm{~mA}$. With these components, the circuit is connected as in FIG. 7, with the smart phone held as a separate unit. Atmel ATMega 16 and use an integrated standard SIM900A. It communicates with PD1 Tx UART with a transmission rate of 9600 bps is used.

\section{B. Installing a CC Cam DVR for Remote Viewing Over Internet}

A few basic things are required to begin:

Live Internet Connection: Directly connecting to the Internet it may come from close to your ISP. In order to be able to provide better, higher speed. However, it is still possible to look at the low-speed connection system, such as from a remote DSL. 
Router: A router is a tool for transferring information among network connections. It allows you to unite several devices to connect to the Internet. Today, many families have a wireless router that can connect to the wireless Internet. The wireless router does not require remote access to the DVR. Some brands of Linksys (Cisco), D-LINK, Netgear, Belkin.

Ethernet Cables: It is commonly used CAT5 (Category 5) cable connected to the Internet. Most DVRs have the ability to remotely access ports that can connect to a CAT5 cable network. The manufacturer also includes a cable with the system, but as long as you do not intend to connect to the router around the DVR, you most if the cable is too short. Connect the modem to the router and you need an Ethernet cable. Routers are usually Ethernet cables too short.

DVR with the ability to be remotely viewed: It does not have to show the ability of all DVR distances. Some video graphs are intended for registration and do not have the ability to connect to them via the Internet. Contact the DVR manufacturer or user controller; make sure you can do this with DVR.

Monitor: When you first make it so that you can view all the settings are set to connect to the DVR, you need to monitor the type. Once these settings are configured, If the remote system is being displayed, the monitor is not necessary.

1. Make sure your modem is connected to the Internet. Normally, there is a light on the front panel of the modem. The status indicator will tell you that it works. Because all the different modems are available from your service provider's information or files. Please go beyond the scope of this article to install the pattern configuration. It is necessary to continue to complete this step.

2. Connect the modem to the router's Internet port. Typically, the router has a port connected to the Internet. These ports are usually far away from the other ports on the back of the router. The port on the back of the router is connected to the Internet. Use a CAT5 cable connection.

3. Put the DVR on a data port on the router. Most routers, at least four ports are equipped with hardware to connect to the Internet. Also with CAT5 cable connection. In the first install, as long as you do not move from the router DVR go, there is no need for length CAT5. After the initial configuration of the built-in DVR cable is complete, you can move the DVR at any time.

Now that you have your hardware and configuration connected, make sure the whole thing is running and running. Then confirm the sub sequential step to arrange your router, DVR and quickly you can see from afar. All of their DVRs have the same interface when you type configure so that they are virtually identical. Other DVRs can have menu that appear a bit dissimilar, other than the parameters are usually the identical. This also applies to the router settings.

\section{Set the DVR}

Connect your DVR to the user name and password provided by the company: If the DVR was earlier configured to use management credentials. Select the Network Setup menu. In this section, you can allocate an IP address to the DVR. It determinate the internal static IP address. (This typically starts with 192.168.XXX.X). I propose you to start with these settings, they can still change later.

a. IP ADDRESS: 192.168.1.110 (This determinate the IP address of the DVR)

b. SUBNETMASK: 255.255.255.0 (Leave it if your system has changed a subnet)

c. GATEWAY: 192.168.1.1 (usually the IP address of the router)

d. TCP: 37777

e. UDP PORT: 37778

f. HTTP PORT: 88 (you can make use of port 80 , but there are internet service providers using port 80. Normally, only block changes will be on the safe side).

Port forwarding on your router: The subsequently article you want to accomplish is port forwarding on your router. This will be necessary to unite your DVR from a remote setting. If you only write your peripheral IP address in your browser, the router doesn't need to recognize which device to access. There might be several super computers and strategy on the network, thus you have to inform the router specifically to assign a type of card to all the ports that you have configured to connect.

\section{ConNected Modules With Working PRINCIPLES}

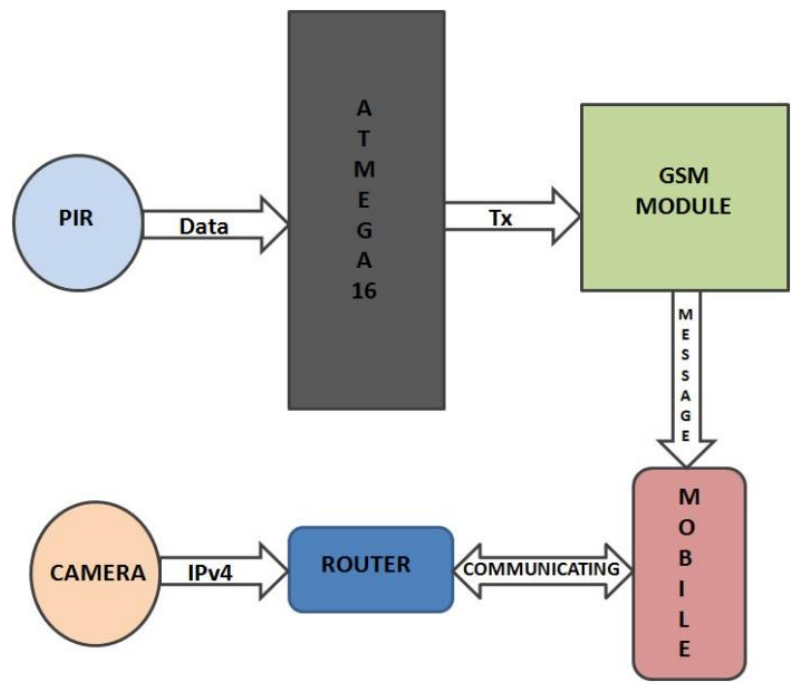

Fig.10. Connectivity of Circuitry Diagram

The first step of this project was to know how many punches per mile PIR mine put out of service. The output of the PIR is a number of the pulses per mile in a 5 volt square wave signal. 


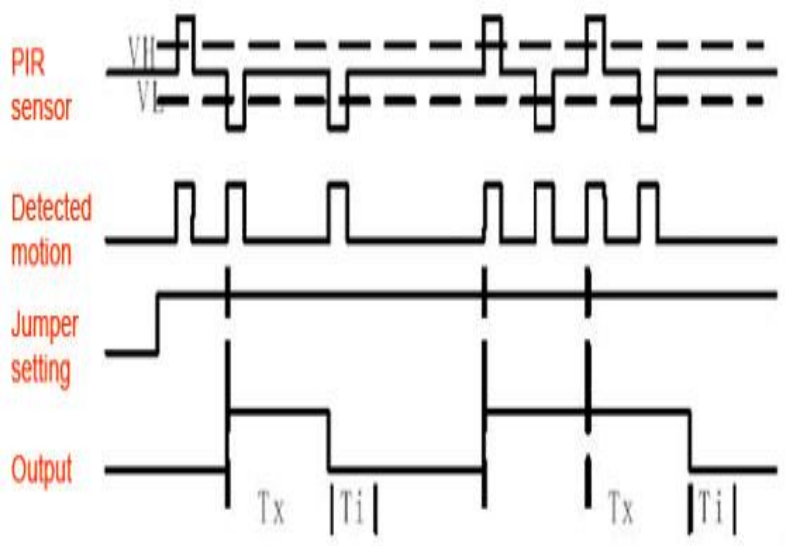

Fig.11. Sensor Input and Output Signal

In PIR sensors, a small trimming potentiometer called TIME. It is a controllable resistor $1 \mathrm{Mohm}$, which is fatherly to a $10 \mathrm{~K}$ series resistor. Moreover C6 is $0.01 \mathrm{uF}$.

$$
\mathrm{Tx}=24576 *(10 \mathrm{~K}+\mathrm{R} \text { time }) * 0.01 \mathrm{uF}
$$

When $\mathrm{R}$ time potentiometer turned counterclockwise completely ( 0 ohms), then

$$
\mathrm{Tx}=24576 * 10 \mathrm{~K} * 0.01 \mathrm{uF} * 2.5 \sec (\text { approx) }
$$

When the potentiometer is fully turned back $\mathrm{R}$ time upward towards the clockwise $1 \mathrm{Meg}$, then

$$
\mathrm{Tx}=24576 * 1010 \mathrm{~K} * 0.01 \mathrm{uF} * 250 \sec (\text { approx })
$$

If $\mathrm{R}$ Time is in the center, it would be concerning 120 seconds (two minutes), so you can customize it as needed.

The data received from sensor is taken in logic ' 1 ' and is stored by using delay time. After it transmit the message using GSM module, the code for this GSM module is given in AT Command. This function informs the user of an SMS message when the movement of a person is detected. This is not only ensuring the safety but also informs the user that "A PERSON IS IN THE ROOM. GO TO THE FOLLOWING LINK 192.168.XX.XX:XXXX".

\section{A PERSON IS IN THE ROOM. GO TO THE FOLLOWING LINK: $192.168 .1,12: 8080$}

Fig.12. Received Message from GSM Module to Mobile

The CC cam and its setup are installed with a particular Static IP address. The DVR is connected to a router with sufficient internet connectivity. Static IP address which is set in the DVR and a specific mobile number is given in coding of microcontroller to generate a message with a particular link to browse as shown in Fig 10. After clicking on the link the browser will be open automatically and start streaming the live video as shown in Fig 11.

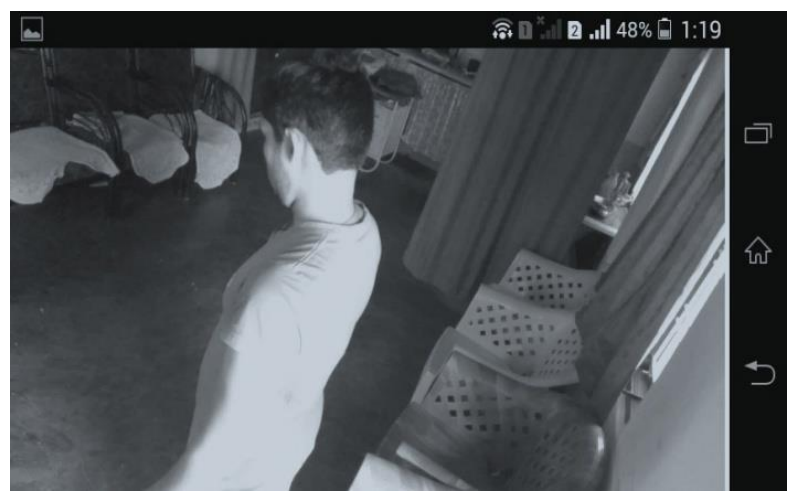

Fig.13. Screenshot of Live Video Streaming

\section{INNOVATED HARDWARE}

The proposed work has implemented in real time. The actual interface is shown in Fig. 12, as is the IoT screenshot of the idea. The proposed work is precise and easy to use.

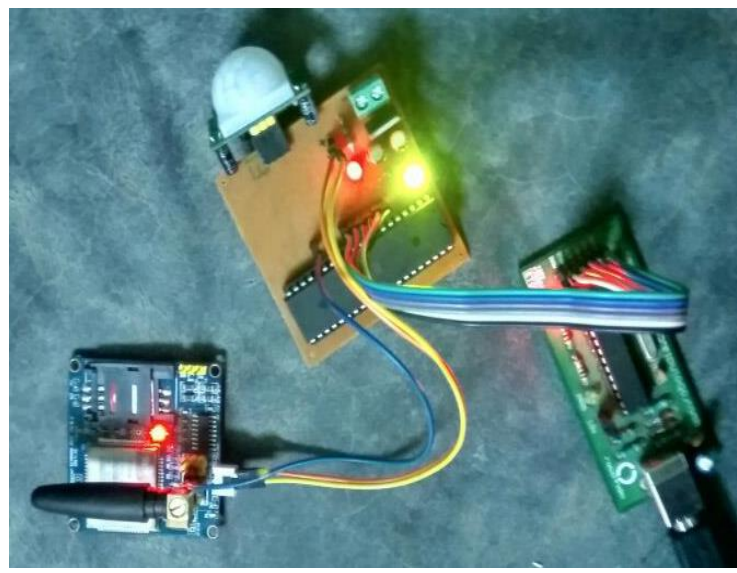

Fig.14. Screenshot of Real Hardware Implement

\section{CONCLUSION AND FutURE SCOPE}

The proposed safety system is successfully tested and implemented in a confined space. This work can also be linked to other fields of application. The power supply circuit from a $5 \mathrm{~V}$ battery will run down at a time, not long circuit the one and after a few hours. This limitation can be solved by connecting the system to the current output from the adapter $5 \mathrm{~V}$ DC $110 \mathrm{~mA}$ (0.55Watt). Similarly, the cam CC also consumes the configuration of energy. This circuit of the safety system can be modified for automatic closing of the door using the above procedure. For high gas for safety reasons manage the level of $\mathrm{O} 2$ and $\mathrm{CO} 2$ to increase or decrease. Otherwise, the entire project can be modified to help us save our home / industries from external threats when we get away from our home / industries. 


\section{ACKNOWLEDGEMENT}

The author of this paper would resembling to expressive great appreciation to ITM University Gwalior, delivering great thanks to CodeVisionAVR and PCB Wizard tools by which apart of work got possible in Embedded System technology.

\section{REFERENCES}

[1] The Study of Access Control for Service-Oriented Computing in Internet of Things Guoping Zhanga, Jing Liub Published Online June 2012 in MECS (http://www.mecs-press.net) DOI:10.5815//ijwmt.2012.03.10.

[2] Myklebust, Gaute. "The AVR Microcontroller and C Compiler Co-Design" Atmel Norway. CiteSeerX 10.1.1.63.1447. Retrieved 2012-09-19.

[3] Real-Time Embedded Systems by Xiaocong Fan.

[4] "Wireless Modules > GSM/GPRS Module > SIM900". SIMCom Wireless Solutions Co.. Retrieved 30. November 2015.

[5] "Wireless Modules > GSM/GPRS > SIM900A". M2Mcom Kft.. Retrieved 04. December 2015.

[6] "How Infrared motion detector components work". Non commercial research page. Glolab Corporation. Retrieved 2013-05-31.

[7] Embedded C, What is Embedded C, Difference between $\mathrm{C}$ and Embedded C Programming, By Akshay Daga, EngineersGarage.

[8] Analysis towards Mobile IPV4 and Mobile IPV6 in Computer Networks Seyedeh Masoumeh Ahmadi Published Online April 2012 in MECS (http://www.mecspress.org/) DOI:10.5815/ijisa.2012.04.05.

[9] Performance Evaluation of IPv4/IPv6 Transition Mechanisms Adira Quintero, Francisco Sans, and Eric Gamess Published Online February 2016 in MECS (http://www.mecs-press.org/) DOI: 10.5815/ijcnis.2016.02.01.

[10] PCB Designers' by Chris Robertson.

[11] "What is portable WiFi router and how to use one". www.worldsim.com. WorldSIM. Retrieved 9 May 2016.

[12] Dempsey, John S. (2008). Introduction to private security. Belmont, CA: Thomson Wadsworth. p. 78. ISBN 9780534558734.

[13] "Internet based CCTV on cloud services" (in Finnish). fennoturvapalvelut. March 27, 2015.

[14] "Basic Home Security and Safety". Secure Home. Retrieved 2016-06-25.
[15] "How To Increase Home Security - Wireless Spy Gadgets". Wireless Spy Gadgets. Retrieved 2016-04-25.

\section{Authors' Profiles}

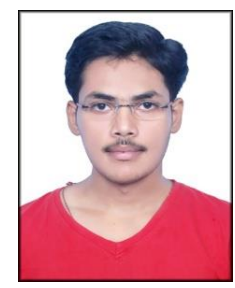

Seelam Vasavi Sai Viswanada Prabhu Deva Kumar born in "Chintalapudi" a city in Andhra Pradesh, India on $15^{\text {th }}$ June 1996. $\mathrm{He}$ is pursuing B.Tech in Electronics \& Communication Engineering from Institute of Technology \& Management University (ITMU), Gwalior in 2014-2018 and area of interest in LED Fabrication and Designing, Embedded Systems \& IoT. Seelam Vasavi Sai Viswanada Prabhu Deva Kumar worked as an Intern in Fuzinix Infotech Pvt. Ltd.

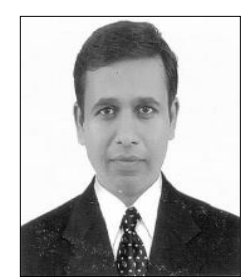

Dr. Shyam Akashe born in "Achalpur" a city in Maharashtra, India on $22^{\text {nd }}$ May 1976. He did M.Tech in Electronics \& Communication Engineering from Rajiv Gandhi Proudyogiki Vishwavidyalaya (RGPV), Bhopal in 2006 and Ph.D Thapar University, Patiala (previously Thapar Institute of Engineering and Technology), Punjab in 2013. Dr. Akashe worked as Assistant Professor at Institute of Technology \& Management (ITM) Gwalior. Currently, he is working as Professor and Post-graduation Coordinator, Electronics \& Communication Engineering department at ITM University Gwalior. Dr. Akashe is authored/co-authored more than 200 research papers in peer reviewed international/national journals and conferences. He has also published 5 patent, 2 copyright, 2 books and 1 chapter. His biography published in Marquis's Who's Who in Engineering Field, USA awarded by Marquis, 2015.

His areas of research are Low Power VLSI Design, Modeling, FinFET based memory design, Circuits for future VLSI Technology, Digital Design and FPGA implementation. He is life member of the Institution of Engineers (IE), Life Member of the Indian Society for Technical Education (IETE), Life Member of the Indian Society for Technical Education (ISTE) and Life Member of the Instrumentation Society of India (ISOI) also he is member of VLSI Society of India (VSI). Dr. Akashe is editorial board member of the IEEE African Journal of Computing \& ICTS (IEEE Nigeria). He is also a reviewer for IEEE Transaction on VLSI Systems, IEEE Transactions on Nanotechnology, International Journal of Electronics (Taylor and Francis), Microelectronics Journal (Elsevier).

How to cite this paper: Seelam Vasavi Sai Viswanada Prabhu Deva Kumar, Shyam Akashe," Implementation of GSM Based Security System with IOT Applications", International Journal of Computer Network and Information Security(IJCNIS), Vol.9, No.6, pp.13-20, 2017.DOI: 10.5815/ijcnis.2017.06.02 